\title{
(6) OPEN ACCESS \\ Mother's education and the risk of preterm and small for gestational age birth: a DRIVERS meta-analysis of 12 European cohorts
}

Milagros Ruiz, ${ }^{1}$ Peter Goldblatt, ${ }^{2}$ Joana Morrison, ${ }^{1}$ Lubomír Kukla, $^{3}$ Jan Švancara, ${ }^{3,4}$ Marjo Riitta-Järvelin, ${ }^{5,7,8,9}$ Anja Taanila, ${ }^{6}$ Marie-Josèphe Saurel-Cubizolles, ${ }^{10}$ Sandrine Lioret, ${ }^{11}$ Chryssa Bakoula, $^{12}$ Alexandra Veltsista, ${ }^{12}$ Daniela Porta, $^{13}$ Francesco Forastiere, ${ }^{13}$ Manon van Eijsden, ${ }^{14}$ Tanja G M Vrijkotte, ${ }^{15}$ Merete Eggesbo, ${ }^{16}$ Richard A White, ${ }^{16}$ Henrique Barros, ${ }^{17,18}$ Sofia Correia, ${ }^{17,18}$ Martine Vrijheid, ${ }^{19,20}$ Maties Torrent, ${ }^{20,21}$ Marisa Rebagliato, ${ }^{20,22}$ Isabel Larrañaga, ${ }^{23,24}$ Johnny Ludvigsson, ${ }^{25}$ Åshild Olsen Faresjö, ${ }^{26}$ Daniel Hryhorczuk, ${ }^{27}$ Youriy Antipkin, ${ }^{28}$ Michael Marmot, ${ }^{1,2}$ Hynek Pikhart $^{1}$

- Additional material is published online only. To view please visit the journal online (http://dx.doi.org/10.1136/jech2014-205387)

For numbered affiliations see end of article.

\section{Correspondence to} Milagros Ruiz, Research Department of Epidemiology and Public Health, University College London, 1-19 Torrington Place, London WC1E 6BT, UK ; milagros.ruiz.10@ucl.ac.uk

Received 16 December 2014 Revised 2 February 2015 Accepted 10 February 2015 Published Online First 24 April 2015

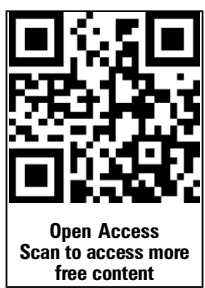

\section{SLinked}

- http://dx.doi.org/10.1136/ jech-2015-205625

\section{CrossMark}

To cite: Ruiz M, Goldblatt P, Morrison J, et al. J Epidemiol Community Health 2015:69:826-833

\section{ABSTRACT}

Background $A$ healthy start to life is a major priority in efforts to reduce health inequalities across Europe, with important implications for the health of future generations. There is limited combined evidence on inequalities in health among newborns across a range of European countries.

Methods Prospective cohort data of 75296 newborns from 12 European countries were used. Maternal education, preterm and small for gestational age births were determined at baseline along with covariate data. Regression models were estimated within each cohort and meta-analyses were conducted to compare and measure heterogeneity between cohorts.

Results Mother's education was linked to an appreciable risk of preterm and small for gestational age (SGA) births across 12 European countries. The excess risk of preterm births associated with low maternal education was 1.48 (1.29 to 1.69) and 1.84 (0.99 to 2.69) in relative and absolute terms (Relative/Slope Index of Inequality, RII/SII) for all cohorts combined. Similar effects were found for SGA births, but absolute inequalities were greater, with an SII score of 3.64 (1.74 to 5.54). Inequalities at birth were strong in the Netherlands, the UK, Sweden and Spain and marginal in other countries studied.

Conclusions This study highlights the value of comparative cohort analysis to better understand the relationship between maternal education and markers of fetal growth in different settings across Europe.

\section{INTRODUCTION}

Prematurity and restricted fetal growth remain a crucial health risk for newborns in Europe. ${ }^{12}$ Poor fetal growth has been linked with not only impaired development throughout early childhood, but evidence supporting the 'fetal origins of adult disease' hypothesis has shown that these at-risk babies are more susceptible to a range of chronic diseases in later adulthood, such as cardiovascular disease, type 2 diabetes mellitus and adiposity, independent of environmental risk factors throughout the life course. ${ }^{34}$
A recent Europe-wide systematic review of child cohort studies has demonstrated the link between maternal education, and the risk of preterm and small for gestational age (SGA) birth, among other markers of fetal growth. ${ }^{5}$ Inequalities have been reported among babies born to mothers with low levels of education in the UK, Denmark, Finland, Norway and Greece. These associations were inconclusive in the Netherlands and Sweden, and negligible in Ireland, Italy, Portugal and Spain. ${ }^{5}$ However, it cannot be readily distinguished whether these reported differences were genuine or artefactual in nature.

Various mechanisms, such as older age at first birth, larger birth intervals, greater use of positive parenting practices, uptake of social and healthcare services, and fewer structural and material barriers, may benefit children of mothers with higher levels of education. These mothers may have an enhanced facility to navigate their familial and socioeconomic environment, and may live in better household-level and neighbourhood-level circumstances favourable to neonatal health. ${ }^{6}$

Studies of mother's education and fetal growth outcomes have been limited to Western and Northern European child cohorts, and contrast the paucity of evidence from Southern and Central/ Eastern Europe. Cross-cohort studies have been restricted to those in Northern areas, and individualcountry studies are marked by differential study design, analysis and control for confounders. ${ }^{5}$ This study aims to comparatively examine the association between mother's education, and preterm and SGA births, among 12 birth cohorts that reflect distinct cultural, geographical and historical settings across Europe. This study has been implemented under the remit of the DRIVERS for Health Equity research programme and in recognition that 'equity from the start' of life is instrumental to reducing health inequalities in Europe. ${ }^{6-8}$

\section{METHODS}

\section{Participants}

Twelve European prospective birth cohorts provide data to this analysis: the European Longitudinal 
Study of Pregnancy and Childhood, Czech Republic (CZELSPAC; $\mathrm{N}=6933)$; the Northern Finland Birth Cohort 1985/1986 Study, Finland (FI-NFBC8586; N=8993); the mother-child Study of Prenatal and Postnatal Determinants of Child Growth, Development, and Health, France (FR-EDEN; $\mathrm{N}=1903$ ); the Greek Birth Cohort, Greece (GR-GBC; $\mathrm{N}=2044)$; the Gene and Environment Prospective Study on Infancy in Italy, Italy (IT-GASPII; $\mathrm{N}=636$ ); the Amsterdam Born Children and their Development Study, the Netherlands (NL-ABCD; $\mathrm{N}=7880$ ); the Norwegian Human Milk Study, Norway (NO-HUMIS; N=2035); the Generation XXI Study, Portugal (PT-G21; N=8330); the Environment and Childhood Project, Spain (ES-INMA; N=2466); the All Babies in Southeast Sweden, Sweden (SE-ABIS; N=15 328); the Millennium Cohort Study, UK (UK-MCS; N=14 630); and the Family and Children of Ukraine Study, Ukraine (UA-FCOU; $N=4118$ ). Details of participating cohorts, including eligibility criteria, are summarised in online supplementary appendix $1 .^{9-25}$ Participants consisted of 75296 babies born between April 1983 and October 2006, and for whom information about maternal education, health and covariate data were available.

\section{Study data}

Study protocols were approved by ethics committees for each cohort. All participating mothers provided consent for themselves and their children. Data on maternal characteristics and health at birth were provided from each cohort in accordance with the ethical procedures approved for each site. Individual-level participant data are defined below.

Mother's education was ascertained during pregnancy or at birth. Years of schooling were available in FR-EDEN, GR-GBC, NL-ABCD, NO-HUMIS and PT-G21. Levels of completed schooling were collected in CZ-ELSPAC, FI-NFBC8586, IT-GASPII, ES-INMA, SE-ABIS and UA-FCOU. Highest obtained qualifications were obtained in UK-MCS. The International Standard Classification of Education (ISCED) is an international categorisation managed by the United Nations Educational, Scientific and Cultural Organization (UNESCO) to facilitate comparison of educational data across countries. The country-specific coding scheme provided by ISCED-1997 was used to classify mothers into high (ISCED 4-6: postsecondary non-tertiary to second stage of tertiary education), medium (ISCED 3: upper secondary education) and low (ISCED 0-2: preprimary to lower secondary or second stage of basic education) categories, using the individual measures of education that were available for each cohort. $^{26}$

Preterm births were defined as live births occurring prior to the gestational age of 37 weeks. In CZ-ELSPAC, FI-NFBC8586, FR-EDEN, NL-ABCD, NO-HUMIS, PT-G21, ES-INMA, SE-ABIS and UA-FCOU, gestational age was based on ultrasound records, if available, or alternatively, using the date of the mother's last menstrual period (LMP). In GR-GBC and IT-GASPII, this was determined only from LMP. The babies' estimated date of delivery was used to determine gestational age in the UK-MCS.

SGA births were defined according to the Alexander fetal growth reference, ${ }^{27}$ which provides expected gender-specific distributions of birth weight (grams) according to gestational age for singleton live births in the USA. Babies were deemed cases if their weight fell below the 10th centile of this national reference projected for their gestational age and gender. ${ }^{27}$ This algorithm has been widely employed in various multicountry studies. ${ }^{28}$ Birth weight was medically assessed in most cohorts, except in SE-ABIS and UK-MCS, which relied on parental reports.
Child sex, maternal age and ethnicity at the time of birth, were potential confounders available in all participating cohorts and thus included in the present study. Mothers belonging to an ethnic minority group participated in 7 out of 12 cohorts. Maternal ethnicity was defined by country of birth in FR-EDEN, IT-GASPII, NL-ABCD and ES-INMA. In NO-HUMIS, mothers were classified as Caucasian, Asian, African, Hispanic, Inuit, Romanian or Oceanian. Mothers in PT-G21 were classified as Portuguese, Portuguese speaking, European migrant, Brazilian, or Other. In UK-MCS, mothers were classified as White, Mixed, Indian, Pakistani/Bangladeshi, Black/Black British or Other.

\section{Statistical analysis}

Analyses were restricted to singletons who made up between $95.9 \%$ and $100 \%$ of the total sample across cohorts. As NO-HUMIS oversampled preterm births in one of their sites, these participants were excluded and analyses were limited to $77.2 \%$ of the original sample.

Study characteristics of each cohort sample were analysed (table 1). The cohort-specific distribution of mother's education was directly age-standardised using the WHO European Standard Population (ESP). ${ }^{29}$ The prevalence of preterm and SGA birth by mother's education was calculated for each cohort, and the $\chi^{2}$ test for trend assessed linearity across educational groups (table 2).

Associations between maternal education and birth outcomes were estimated to infer relative and absolute socioeconomic inequalities in each cohort sample using the Relative (and Slope) Index of Inequality (RII/SII). The regression-based indices consider both the size and distribution of socioeconomic groups across the population, by evaluating morbidity risk according to the specific proportions of the population within the socioeconomic hierarchy. Given the extent of cohort differences in maternal education, these indices account for such differences as a source of variation in the magnitude of health inequalities, and facilitate comparison of estimates between cohorts. ${ }^{30-32}$ The RII is a summary measure of relative inequality, defined as the prevalence ratio of the child outcome between children at the lowest and those at the highest end of the maternal education hierarchy. The SII is the corresponding measure of absolute inequality, defined as the prevalence difference of the child outcome between the two ends of said hierarchy. A RII score greater than 1 (and a SII score greater than 0) indicates the presence of inequality between low and high positions. For both indices, higher scores denote a larger magnitude of inequality.

Generalised linear models were performed to obtain the RII and SII by, respectively, specifying a logarithmic or an identity link function. Educational categories were ranked from high to low and were each assigned a value between 0 and 1 , based on the cumulative percentage of the midpoint of the ranges observed for each cohort. ${ }^{33}$ Models were adjusted for child sex, maternal age and ethnicity, as appropriate, for each outcome. All analyses of the UK-MCS employed survey weights to account for the cohort's sampling design. ${ }^{24}$

Cohort-specific scores were pooled together to obtain mean RII and SII scores at the European level using random effects meta-analysis procedures, given between-cohort heterogeneity (figures 1 and 2). The extent of heterogeneity was tested using the $\mathrm{Q}$ test and the $\mathrm{I}^{2}$ measure, ${ }^{34}$ which confirmed the a priori expectation of heterogeneity between cohorts not attributable to within-cohort variation. Sensitivity analyses using fixed-effects meta-analysis techniques yielded estimates similar to those obtained by random-effects methods. All analyses were carried out using Stata V.13 (Stata Corp, College Station, Texas, USA). 
Table 1 Overview and study characteristics of analytic cohort samples

\begin{tabular}{|c|c|c|c|c|c|c|c|c|c|c|c|c|}
\hline $\begin{array}{l}\text { Country* } \\
\text { Birth cohort }\end{array}$ & $\begin{array}{l}\text { FR } \\
\text { EDEN }\end{array}$ & $\begin{array}{l}\mathrm{NL} \\
\mathrm{ABCD}\end{array}$ & $\begin{array}{l}\text { UK } \\
\text { MCSt }\end{array}$ & $\begin{array}{l}\text { CZ } \\
\text { ELSPAC }\end{array}$ & $\begin{array}{l}\text { UA } \\
\text { FCOU }\end{array}$ & $\begin{array}{l}\text { FI } \\
\text { NFBC8586 }\end{array}$ & $\begin{array}{l}\text { NO } \\
\text { HUMIS }\end{array}$ & $\begin{array}{l}\text { SE } \\
\text { ABIS }\end{array}$ & $\begin{array}{l}\text { GR } \\
\text { GBC }\end{array}$ & $\begin{array}{l}\text { IT } \\
\text { GASPII }\end{array}$ & $\begin{array}{l}\text { PT } \\
\text { G21 }\end{array}$ & $\begin{array}{l}\text { ES } \\
\text { INMA }\end{array}$ \\
\hline Year(s) of birth & $2003 / 2006$ & $2003 / 2004$ & $2001 / 2002$ & 1991/1992 & 1993/1996 & 1985/1986 & $2003 / 2006$ & 1997/1999 & 1983 & $2003 / 2004$ & $2005 / 2006$ & $1997 / 2005$ \\
\hline $\mathrm{N}$ at birth $¥$ & 1903 & 7880 & 14630 & 6933 & 4118 & 8993 & 2035 & 15328 & 2044 & 636 & 8330 & 2466 \\
\hline Per cent boys (N) & $52.6(1000)$ & $50.4(3969)$ & $51.0(7467)$ & $51.8(3593)$ & $52.7(2170)$ & $51.3(4617)$ & $52.4(1066)$ & $51.7(7920)$ & $46.6(953)$ & $50.8(323)$ & $51.1(4259)$ & $51.6(1272)$ \\
\hline Per cent girls (N) & $47.5(903)$ & $49.6(3911)$ & $49.0(7163)$ & $48.2(3340)$ & $47.3(1948)$ & $48.7(4376)$ & 47.6 (969) & $48.3(7408)$ & $53.4(1091)$ & $49.2(313)$ & $48.9(4071)$ & $48.4(1194)$ \\
\hline \multicolumn{13}{|c|}{ Mother's characteristics at time of birth } \\
\hline \multicolumn{13}{|l|}{ Educational classification, \%§ } \\
\hline High & 51.7 & 36.5 & 21.1 & 25.5 & 33.6 & 17.5 & 70.7 & 26.7 & 10.6 & 27.4 & 14.8 & 21.9 \\
\hline Medium & 36.1 & 28.1 & 41.3 & 38.2 & 61.8 & 51.1 & 23.6 & 25.8 & 20.5 & 47.5 & 19.4 & 34.7 \\
\hline Low & 12.2 & 35.3 & 37.6 & 36.3 & 4.7 & 31.4 & 5.7 & 47.5 & 68.9 & 25.1 & 65.8 & 43.3 \\
\hline Age (years), mean & 29.5 & 30.7 & 28.9 & 25.3 & 23.8 & 27.8 & 29.9 & 30.2 & 25.8 & 32.9 & 29.0 & 31.4 \\
\hline Ethnic minority, \% & 4.0 & 29.7 & 10.8 & 1.1 & 0 & 0 & 11.4 & 0 & 0 & 6.0 & 3.3 & 6.0 \\
\hline \multicolumn{13}{|l|}{ Birth outcomes } \\
\hline Preterm birth, \% & 5.8 & 5.7 & 6.8 & 1.7 & 5.8 & 4.4 & 3.4 & 3.7 & 4.7 & 4.4 & 7.5 & 4.6 \\
\hline Small for gestational age, $\%$ & 11.4 & 7.4 & 10.9 & 11.7 & 7.8 & 4.8 & 5.4 & 4.6 & 8.5 & 9.1 & 12.7 & 15.3 \\
\hline
\end{tabular}

*Country abbreviations represent France (FR), the Netherlands (NL), the UK (UK), the Czech Republic (CZ), Ukraine (UA), Finland (FI), Norway (NO), Sweden (SE), Greece (GR), Italy (IT), Portugal (PT) and Spain (ES).

tDescriptive data of the MCS sample use survey weights to account for the cohort study's sampling design.

ҒApproximate sample sizes from which health outcome data for the present analysis were ascertained.

$\S$ Educational levels in each country have been age-standardised to the WHO European Standard Population.

MCS, Millennium Cohort Study.

Table 2 Cohort-specific prevalence of preterm and SGA births by maternal education

\begin{tabular}{|c|c|c|c|c|c|c|c|c|c|c|c|c|}
\hline $\begin{array}{l}\text { Country* } \\
\text { Birth cohort }\end{array}$ & $\begin{array}{l}\text { FR } \\
\text { EDEN }\end{array}$ & $\begin{array}{l}\text { NL } \\
\text { ABCD }\end{array}$ & $\begin{array}{l}\text { UK } \\
\text { MCSt }\end{array}$ & $\begin{array}{l}\text { CZ } \\
\text { ELSPAC }\end{array}$ & $\begin{array}{l}\text { UA } \\
\text { FCOU }\end{array}$ & $\begin{array}{l}\text { FI } \\
\text { NFBC8586 }\end{array}$ & $\begin{array}{l}\text { NO } \\
\text { HUMIS }\end{array}$ & $\begin{array}{l}\text { SE } \\
\text { ABIS }\end{array}$ & $\begin{array}{l}\text { GR } \\
\text { GBC }\end{array}$ & $\begin{array}{l}\text { IT } \\
\text { GASPII }\end{array}$ & $\begin{array}{l}\text { PT } \\
\text { G21 }\end{array}$ & $\begin{array}{l}\text { ES } \\
\text { INMA }\end{array}$ \\
\hline \multicolumn{13}{|c|}{ Preterm birth, \% } \\
\hline High & 5.2 & 4.9 & 6.0 & 1.7 & 4.4 & 4.0 & 5.9 & 3.3 & 8.9 & 4.4 & 7.4 & 3.3 \\
\hline Medium & 6.0 & 6.3 & 6.9 & 1.1 & 6.1 & 4.0 & 9.2 & 3.5 & 3.3 & 3.4 & 6.4 & 4.3 \\
\hline Low & 8.6 & 7.0 & 8.2 & 1.5 & 7.3 & 5.4 & 9.7 & 4.3 & 4.7 & 8.3 & 8.1 & 6.3 \\
\hline$p$ for trend & 0.146 & 0.001 & 0.014 & 0.884 & 0.064 & 0.021 & 0.009 & 0.012 & 0.178 & 0.331 & 0.192 & 0.005 \\
\hline \multicolumn{13}{|l|}{ SGA birth, \% } \\
\hline High & 9.7 & 5.4 & 7.6 & 10.5 & 6.7 & 3.9 & 5.9 & 4.1 & 8.9 & 6.6 & 11.8 & 11.9 \\
\hline Medium & 13.5 & 9.1 & 11.1 & 10.9 & 7.7 & 4.7 & 7.5 & 4.2 & 8.2 & 10.8 & 11.4 & 15.3 \\
\hline Low & 12.9 & 10.8 & 17.2 & 11.2 & 12.2 & 5.3 & 8.1 & 5.3 & 8.4 & 9.5 & 13.7 & 18.5 \\
\hline$p$ for trend & 0.026 & $<0.001$ & $<0.001$ & 0.605 & 0.024 & 0.036 & 0.165 & 0.003 & 0.912 & 0.203 & 0.012 & $<0.001$ \\
\hline
\end{tabular}

*Country abbreviations represent France (FR), the Netherlands (NL), the UK (UK), the Czech Republic (CZ), Ukraine (UA), Finland (FI), Norway (NO), Sweden (SE), Greece (GR), Italy (IT), Portugal (PT) and Spain (ES).

tDescriptive data of the MCS sample use survey weights to account for the cohort study's sampling design.

MCS, Millennium Cohort Study; SGA, small for gestational age. 


\section{RESULTS}

Mothers were primarily representative of the ethnic majority of their cohort, and aged between 23.8 and 31.4 years at the time of birth (table 1). Substantially more mothers with low education were present in ES-INMA (44\%), and in GR-GBC and PT-G21 $(65 \%>)$. Conversely, the proportion of lowly educated mothers were very few $(<5 \%)$ in UA-FCOU. Mothers with high education were greater $(50 \%>)$ in FR-EDEN and NO-HUMIS and lower

A

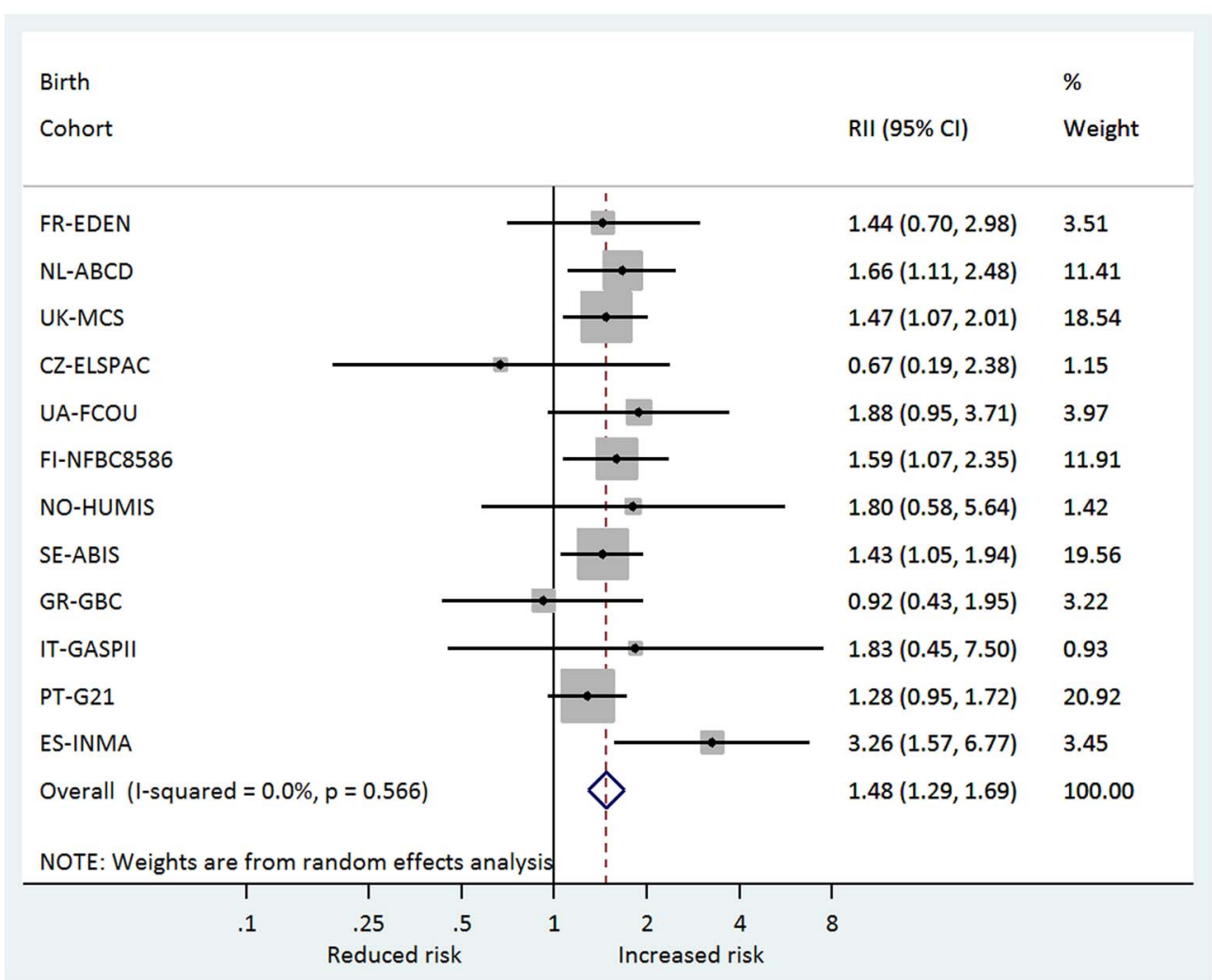

B

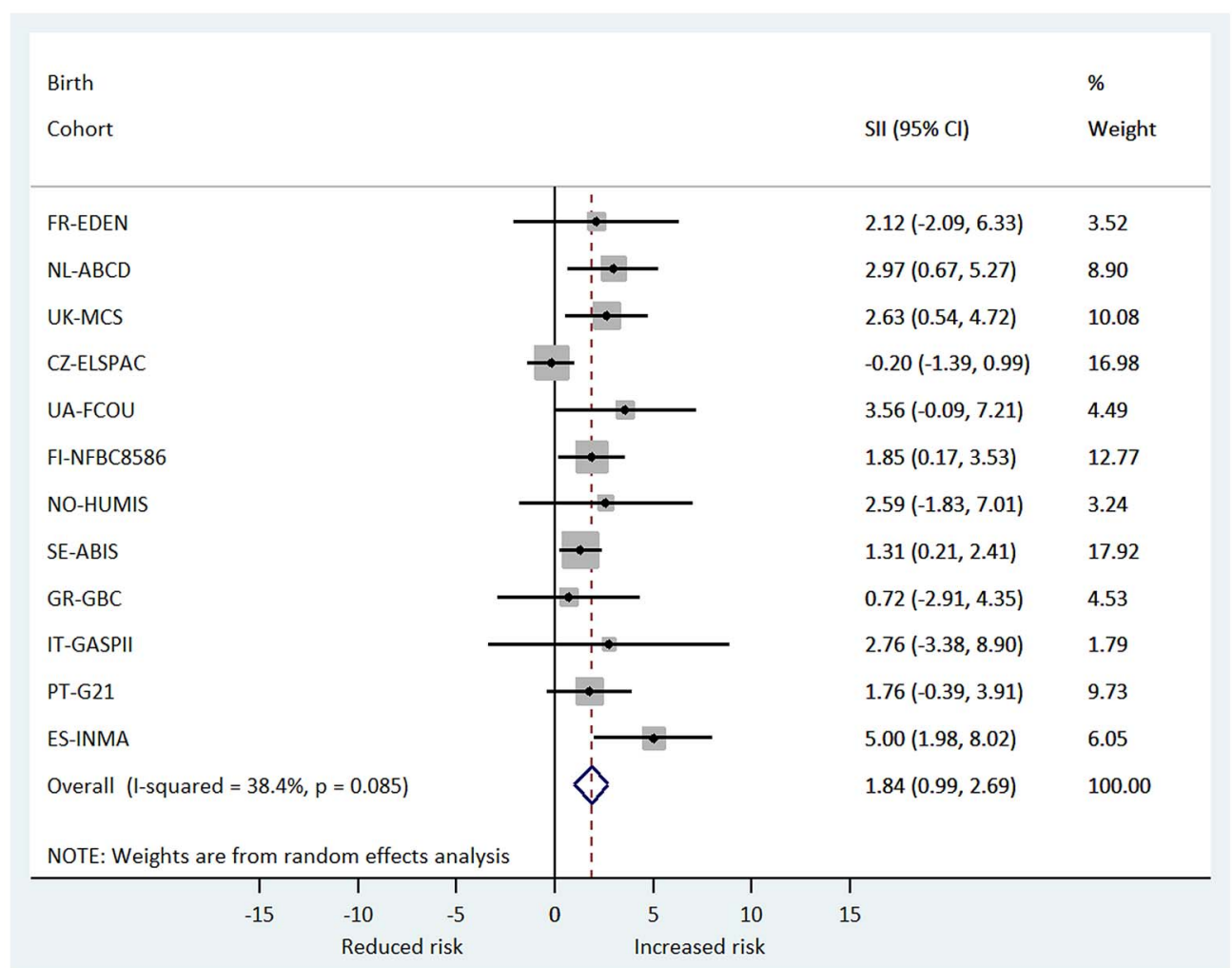

Figure 1 (A/B) - Cohort-specific, and pooled Relative/Slope Index of Inequality, RII and SII scores, in the meta-analysis of the association between maternal education and preterm birth. 


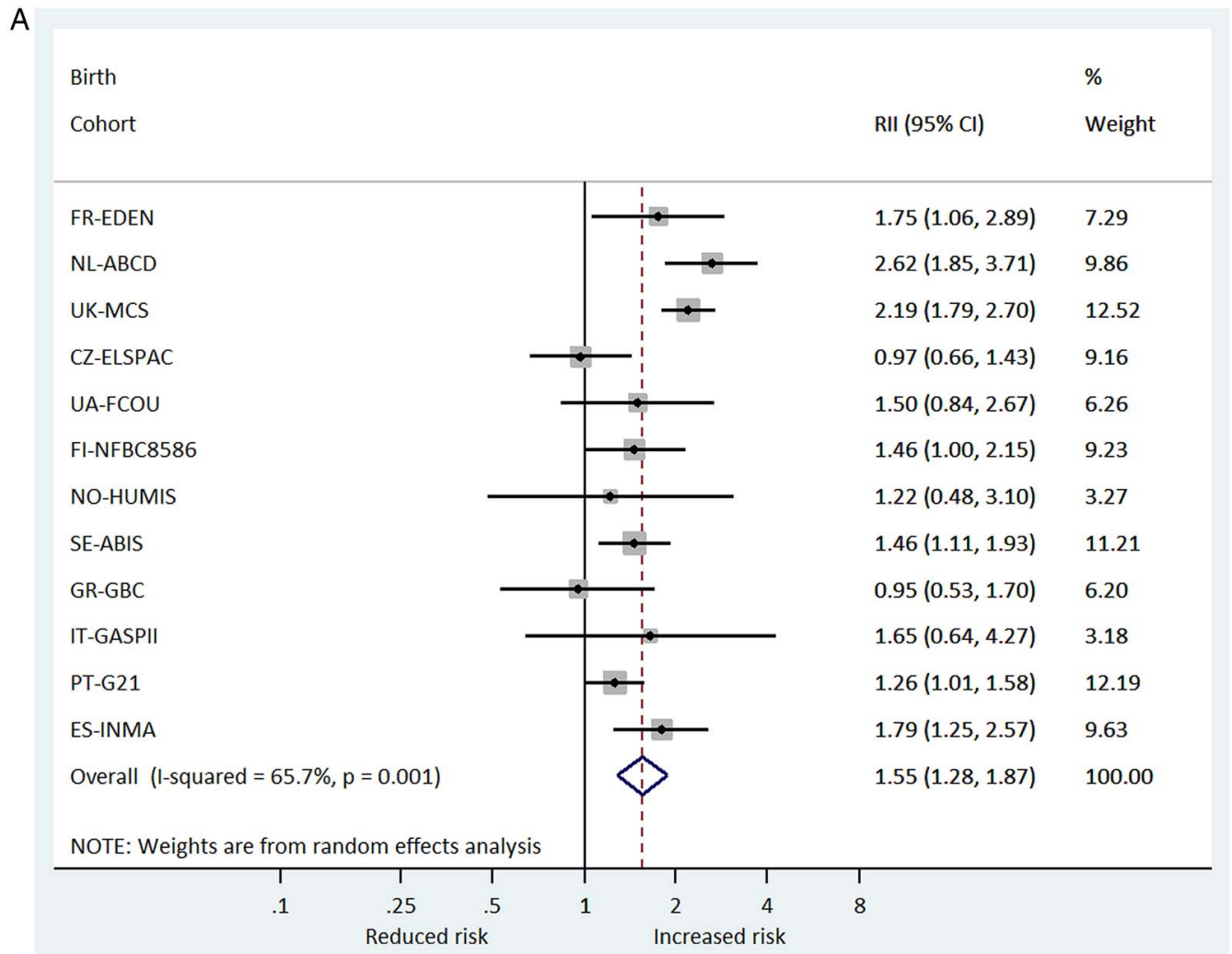

B

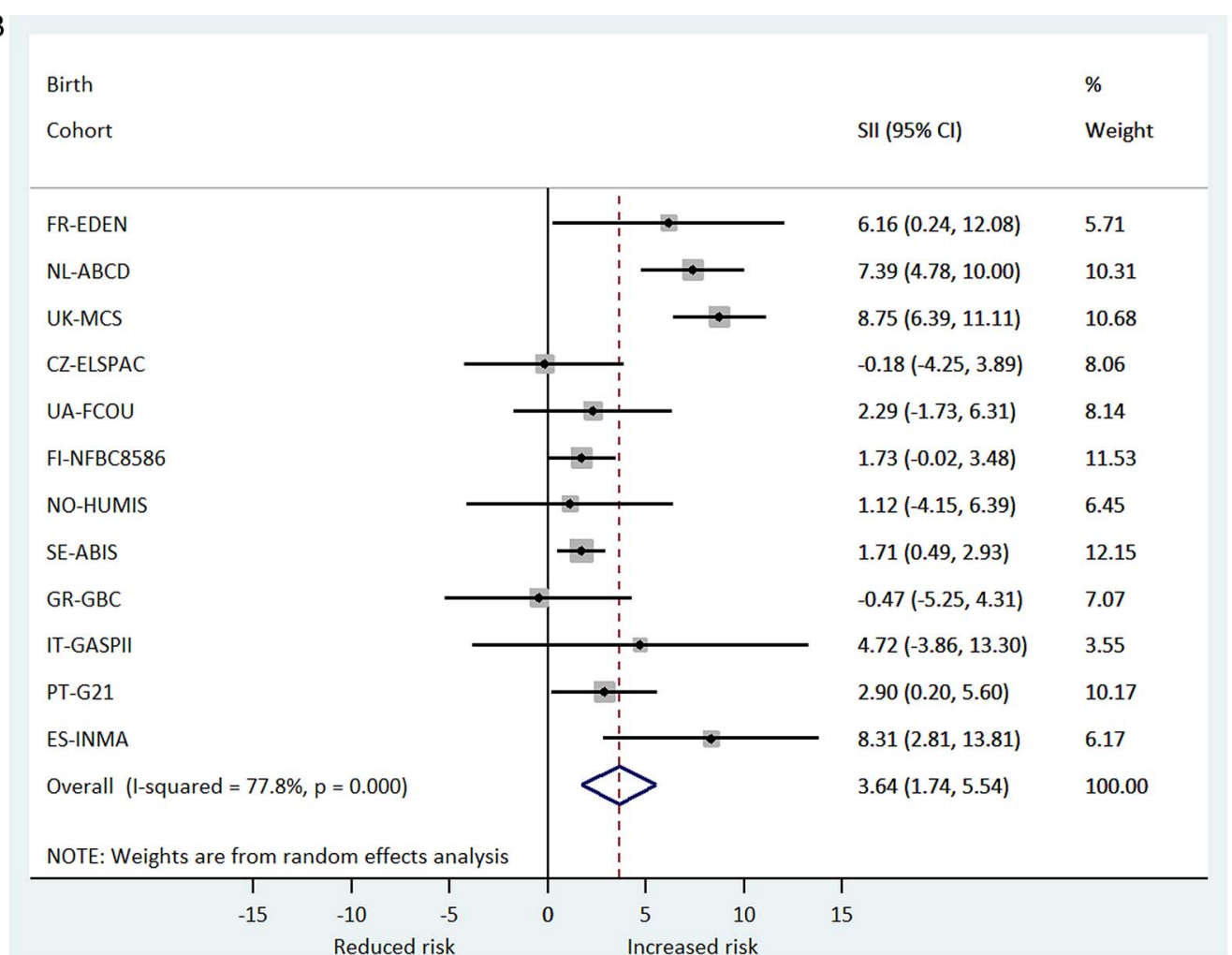

Figure 2 (A/B) —Cohort-specific, and pooled Relative/Slope Index of Inequality, RII and SII scores, in the meta-analysis of the association between maternal education and small for gestational age birth.

elsewhere. Preterm births ranged from 1.7\% in CZ-ELSPAC to $6.8 \%$ in UK-MCS. SGA births showed wider differences, as prevalence was lowest in FI-NFBC8586, NO-HUMIS and SE-ABIS (4.6-5.4\%), and highest in ES-INMA and PT-G21 (12.7-15.3\%).
Educational gradients in preterm and SGA births were palpable in NL-ABCD, UK-MCS, FI-NFBC8586, SE-ABIS and ES-INMA (table 2). Similar gradients were found in prevalent SGA, but not in preterm births, in FR-EDEN, UA-FCOU and 
PT-G21. The gradient in NO-HUMIS was apparent for preterm birth only. Trends were inconclusive in CZ-ELSPAC, GR-GBC and IT-GASPII for either birth outcome.

Figure 1 displays the magnitude of inequalities in preterm birth. Cohort-specific scores ranged from 0.67 in CZ-ELSPAC to 3.26 in ES-INMA, and from -0.20 to 5.00 in the aforementioned cohorts, respectively, for the RII and SII. The mean RII and SII score indicated a heightened relative and absolute risk, in preterm birth, of $48 \%$ and $84 \%$ for babies born to mothers with low education (RII $=1.48,95 \%$ CI 1.29 to 1.69 , SII $=1.84$, $95 \%$ CI 0.99 to 2.69 ) combined for all cohorts. No observed heterogeneity was found for relative inequalities, as shown by the Q test of $9.61(\mathrm{df}=11, \mathrm{p}=0.566)$ and the $\mathrm{I}^{2}$ statistic of $0 \%$. Low to moderate heterogeneity was estimated for absolute inequalities given the $\mathrm{I}^{2}$ statistic of $38.4 \%$, although the Q test of 17.85 did not approach significance $(\mathrm{df}=11, \mathrm{p}=0.085)$. Cohort-specific findings revealed significant associations in NL-ABCD, UK-MCS, FI-NFBC8586, SE-ABIS and ES-INMA, but not in FR-EDEN, UA-FCOU, NO-HUMIS, IT-GASPII and PT-G21. A positive, although insignificant, association between high maternal education and greater risk of preterm birth was observed in CZ-ELSPAC and PT-G21.

Equivalent estimates of inequalities in SGA births ranged from 0.97 in CZ-ELSPAC to 2.62 in NL-ABCD, and from -0.47 in GR-GBC to 8.75 in UK-MCS (figure 2). The mean RII and SII score pooled for all cohorts yielded a $55 \%$ increase in relative risk and a $3.6 \%$ excess in absolute prevalence in SGA births among babies born to mothers with low education (RII $=1.55,95 \%$ CI 1.28 to $1.87, \mathrm{SII}=3.64,95 \%$ CI 1.74 to 5.54). Unlike the preterm birth meta-analysis, these results emphasise moderate to high between-cohort heterogeneity in relative and in absolute inequalities. This is shown by the $\mathrm{Q}$ test of $32.06(\mathrm{df}=11, \mathrm{p}=0.001)$ and $49.64(\mathrm{df}=11, \mathrm{p} \leq 0.001)$, in addition to the $\mathrm{I}^{2}$ statistic of $65.7 \%$ and $77.8 \%$, respectively, for the RII and SII scores. Evidence from FR-EDEN, NL-ABCD, UK-MCS, SE-ABIS, PT-G21 and ES-INMA indicated strong inequalities in SGA births among these cohorts, but were weak in UA-FCOU, FI-NFBC8586, NO-HUMIS and IT-GASPII. Positive associations in CZ-ELSPAC and GR-GBC were not statistically significant.

Cohort-specific inequalities generally corresponded across the two examined birth outcomes, with a few exceptions. Mothers with low education in FR-EDEN and PT-G21 were at a greater risk to have babies born SGA, but not preterm. In FI-NFBC8586, inequalities were present in preterm, but not in SGA births.

\section{DISCUSSION}

This is the largest European study of educational inequalities in newborn health, which establishes the broad influence of mother's education on the likelihood of preterm and SGA births. Cohort-specific inequalities were consistently apparent in the Netherlands, the UK, Sweden and Spain, but negligible in the Czech Republic, Ukraine, Norway, Greece and Italy. Inequalities in preterm births alone were observed in Finland. Low maternal education was associated with a greater risk of SGA but not preterm births in France and Portugal.

Routine collection of mother's education data enabled us to carry out this large-scale study. Reliance of mother's education over other family-level and neighbourhood-level socioeconomic markers may not fully expose inequalities in preterm and SGA risk. It is plausible that negligible inequalities reported in this study may be pronounced by another marker representative of a newborn's social environment. Nonetheless, education is more amenable for cross-country research than other markers of socioeconomic position, such as occupational class and does not significantly bias our findings. ${ }^{35}{ }^{36}$ Available for all women including working mothers and full-time homemakers outside of the labour market, ${ }^{37}$ education is more likely to remain constant during the child-rearing period and is less prone to misreporting than income or wealth. ${ }^{38}$

Bias due to the country-specific ISCED classification may falsely enhance between-country differences, but would likely be random and unrelated to the underlying associations between maternal education and newborn health within countries. Mothers with low education were remarkably few in Norway and Ukraine but high in Greece. These proportions matched closely with national averages reported in the European Health for All Database (HFA-DB) during the years in which cohorts conducted their baseline assessments, and were thus representative of their countries in this respect. ${ }^{29}$

Two birth outcomes measured by trained personnel using internationally recognised definitions were studied. SGA births defined according to a common reference ${ }^{27}$ may differentially influence prevalence estimates due to diverse fetal growth patterns between cohorts. Applying cohort-specific references may account for these, but misclassification may also occur due to other differences in cohort procedures. ${ }^{39}$ Following methods commonly used in various European data sources, calculation of gestational age among participating cohorts was varied to some extent, but there is no approved best practice for assessing gestational age in the literature. ${ }^{40}$

While the multipurpose nature of participating cohorts with unique study designs, selected participants and periods covered may pose a methodological limitation, the cohorts reflect among the most current of child birth cohorts across Europe available for this study. Adjustment procedures do not eliminate the role of residual confounding by factors that were not available for this analysis. Completeness of gestational age and birth weight data was generally achieved in all cohorts, as these data are customarily collected for all newborns. However, data were more commonly missing among babies born to mothers with low education in the Czech Republic, Greece, Portugal and Sweden. A reverse pattern was found in France, Italy, the Netherlands and Spain. No evident pattern of missingness by maternal education was found in the UK or Ukraine.

This meta-analysis leads us to consider whether we may anticipate differences in the relationship between maternal education and health at birth, both between and within the selected 12 European countries. Very few multicountry studies on educational inequalities in preterm and SGA births have been performed across Europe; and have been limited to Northern and Western populations. ${ }^{5}$ Notwithstanding, extensive research on adult health inequalities has elucidated how national structures with particular income and welfare contexts, healthcare and social care systems, among others, differ between countries of Europe. These may also differentially impact inequalities in newborn health.

Country differences reported in this meta-analysis may be consequent to national differences in maternal education, the overall level of newborn health, as well as plausible mediating pathways, such as women's participation in the labour market, gender-specific income and work life-related programmes, social norms that influence women's health and child-rearing behaviours and family arrangements, among other societal, political and cultural factors. While data on such factors are not readily available for this investigation, future DRIVERS work will comprise comparative analysis to assess potential mediators in the relationship between maternal education, and preterm and SGA 
birth. Such evidence may shed light on country differences observed in different European settings.

\section{CONCLUSIONS}

Poor health at birth is greater among babies of mothers with low education across all cohorts combined from 12 European countries. Inequalities were strongest in the Netherlands, the UK, Sweden and Spain. The study illustrates the need to improve newborn health and to reduce these inequalities across distinct European populations. The period of infancy is crucial to children's immediate and subsequent health and development, and in generating social and health inequalities across the life course and between generations.

\section{What is already known on this subject}

Low levels of maternal education have been found to be associated with adverse neonatal outcomes in offspring. However, few studies have systematically assessed this at the European level, and it is unclear whether associations are consistent between European countries.

\section{What this study adds}

This study showed that children born to mothers with low education were more likely to be born preterm and small for their gestational age at the regional level, but associations were not consistent between selected cohorts in 12 European countries.

\section{Author affiliations}

${ }^{1}$ Research Department of Epidemiology and Public Health, University College London, London, UK

${ }^{2}$ Research Department of Epidemiology and Public Health, UCL Institute of Health Equity, University College London, London, UK

${ }^{3}$ Faculty of Science, Research Centre for Toxic Compounds in the Environment (RECETOX), Masaryk University, Brno, Czech Republic

${ }^{4}$ Faculty of Medicine, Institute of Biostatistics and Analyses, Masaryk University, Brno, Czech Republic

${ }^{5}$ Department of Epidemiology and Biostatistics, MRC Health Protection Agency (HPE), Centre for Environment and Health, School of Public Health, Imperial College London, London, UK

${ }^{6}$ Biocenter Oulu, University of Oulu, Oulu, Finland

${ }^{7}$ Unit of Primary Care, Oulu University Hospital, Oulu, Finland

${ }^{8}$ Center for Life Course Epidemiology, Faculty of Medicine, University of Oulu, Oulu, Finland

${ }^{9}$ Institute of Health Sciences, University of Oulu, Oulu, Finland

${ }^{10}$ Institut National de la Santé et de la Recherche Médicale (INSERM) UMR 1153, Obstetrical, Perinatal and Pediatric Epidemiology Research Team (Epopé), Center for Epidemiology and Statistics, Sorbonne Paris Cité, DHU Risks in Pregnancy, Paris Descartes University, Paris, France

${ }^{11}$ Institut National de la Santé et de la Recherche Médicale (INSERM) UMR 1153 Early Origin of the Child's Health and Development Team (ORCHAD), Center for Epidemiology and Statistics, Sorbonne Paris Cité, Paris Descartes University, Paris, France

${ }^{12}$ First Department of Paediatrics, University of Athens, Aghia Sophia Children's Hospital, Athens, Greece

${ }^{13}$ Department of Epidemiology of the Lazio Regional Health System, Rome, Italy

${ }^{14}$ Department of Epidemiology and Health Promotion, Public Health Service of Amsterdam, Amsterdam, The Netherlands

${ }^{15}$ Department of Public Health, Academic Medical Center, University of Amsterdam, Amsterdam, The Netherlands

${ }^{16}$ Department of Genes and Environment, Norwegian Institute of Public Health, Oslo, Norway
${ }^{17}$ Department of Clinical Epidemiology, Predictive Medicine and Public Health, University of Porto Medical School, Porto, Portugal

${ }^{18}$ EPIUnit - Institute of Public Health, University of Porto, Porto, Portugal ${ }^{19}$ Center for Research in Environmental Epidemiology (CREAL), Barcelona, Spain

${ }^{20}$ Spanish Consortium for Research on Epidemiology and Public Health (CIBERESP), Barcelona, Spain

${ }^{21}$ IB-Salut Menorca Health Area, Balearic Islands, Spain

${ }^{22}$ Departamento de Medicina, Universitat Jaume I, Castellon, Spain

${ }^{23}$ Public Health Department of Gipuzkoa, Gipuzkoa, Spain

${ }^{24}$ BIODONOSTIA Health Research Institute, San Sebastian, Spain

${ }^{25}$ Division of Pediatrics, Department of Clinical and Experimental Medicine, Linköping University, Linköping, Sweden

${ }^{26}$ Department of Medicine and Health Sciences, Linköping University, Linköping, Sweden

${ }^{27}$ Center for Global Health, University of Illinois College of Medicine, Chicago, Illinois, USA

${ }^{28}$ Institute of Pediatrics, Obstetrics, and Gynecology, Kyiv, Ukraine

Twitter Follow Milagros Ruiz at @MilagrosRuizLu

Acknowledgements The authors are grateful to participating members of the European birth cohort studies included in the present paper. The authors would like to acknowledge Marie-Aline Charles, Anne Forhan and Barbara Heude from the EDEN Mother-Child Cohort Study. In addition, the authors would like to acknowledge Alexander Zvinchuk for his work on preparing the UA-FCOU data for this collaborative paper. He is the Director of the Louis Hamilton Data Management Center in Ukraine, which is housed in the Institute of Pediatrics, Obstetrics and Gynecology in Kyiv. The authors are very grateful for constructive review to an earlie version of the manuscript given by the following members of the DRIVERS Consortium: Linden Farrer, EuroHealthNet; Olle Lundberg, Stockholm University; and Diego Montano and Johannes Siegrist, University of Duesseldorf.

Contributors MR designed the study, carried out all analyses, drafted the initial manuscript and approved the final manuscript as submitted. HP conceptualised and designed the study, oversaw all analyses and approved the final manuscript as submitted. PG, JM and MM reviewed and revised the manuscript, and approved the final manuscript as submitted. LK, JS, MR-J, AT, M-JS-C, SL, CB, AV, DP, FF, MvE, TGMV, ME, RAW, HB, SC, MV, MT, MR, IL, JL, AOF, DH and YA procured and coordinated data for this study, critically reviewed the manuscript and approved the final manuscript as submitted.

Funding All phases of this study were supported by a European Union's Seventh Framework Programme grant, 278350, as part of The Determinants to Reduce Health Inequity Via Early Childhood, Realising Fair Employment, and Social Protection (DRIVERS) research programme. The Czech ELSPAC Study was supported by the Ministry of Education of the Czech Republic (LM2011028, L01214) and the Grant Agency of the Masaryk University (MUNI/M/1075/2013). The Northern Finland Birth Cohort (NFBC8586) received financial support from the Academy of Finland, Biocenter, University of Oulu, Finland, the European Commission (EURO-BLCS, Framework 5 award QLG1-CT-2000-01643), EU FP7 EurHEALTHAgeing -277849, the Medical Research Council, UK ( PrevMetSyn/SALVE) and the MRC Centenary Early Career Award.

Competing interests None declared.

Ethics approval Study protocols of participating birth cohorts were approved by ethics committees for each respective cohort.

Provenance and peer review Not commissioned; externally peer reviewed.

Open Access This is an Open Access article distributed in accordance with the Creative Commons Attribution Non Commercial (CC BY-NC 4.0) license, which permits others to distribute, remix, adapt, build upon this work non-commercially, and license their derivative works on different terms, provided the original work is properly cited and the use is non-commercial. See: http://creativecommons.org/ licenses/by-nc/4.0/

\section{REFERENCES}

1 EURO-Peristat. European Perinatal Health Report. 2010.

2 Beck S, Wojdyla D, Say L, et al. The worldwide incidence of preterm birth: a systematic review of maternal mortality and morbidity. Bull World Health Organ 2010;88:31-8.

3 Gluckman PD, Hanson MA, Cooper $C$, et al. Effect of in utero and early-life conditions on adult health and disease. N Engl J Med 2008;359:61-73.

4 Barker DJ, Gluckman PD, Godfrey KM, et al. Fetal nutrition and cardiovascular disease in adult life. Lancet 1993;341:938-41.

5 Pillas D, Marmot M, Naicker $K$, et al. Social inequalities in early childhood health and development: a European-wide systematic review. Pediatr Res 2014;76:418-24

6 WHO Regional Office for Europe. Review of social determinants and the health divide in the WHO European Region: Final Report. 2013 
7 DRIVERS for Health Equity (FP7). http://health-gradient.eu/

8 Commission on Social Determinants of Health, WHO. Closing the gap in a generation: health equity through action on the social determinants of health. Geneva: World Health Organization (WHO), 2008.

9 Henderson AJ, Sherriff A, Northstone K, et al. Pre- and postnatal parental smoking and wheeze in infancy: cross cultural differences. Avon Study of Parents and Children (ALSPAC) Study Team, European Longitudinal Study of Pregnancy and Childhood (ELSPAC) Co-ordinating Centre. Eur Respir J 2001;18:323-9.

10 Kukla L, Hruba D, Tyrlik M. Influence of prenatal and postnatal exposure to passive smoking on infants' health during the first six months of their life. Cent Eur J Public Health 2004;12:157-60.

11 Jarvelin MR, Elliott P, Kleinschmidt I, et al. Ecological and individual predictors of birthweight in a northern Finland birth cohort 1986. Paediatr Perinat Epidemio 1997;11:298-312.

12 The University of Oulu. Northern Finland Birth Cohorts, NFBC. http://www.oulu.fi/ nfbc/node/20139 (accessed 27 Jan 2015).

13 Drouillet $\mathrm{P}$, Forhan A, De Lauzon-Guillain B, et al. Maternal fatty acid intake and fetal growth: evidence for an association in overweight women. The 'EDEN mother-child' cohort (study of pre- and early postnatal determinants of the child's development and health). Br J Nutr 2009;101:583-91.

14 EDEN VJF INSERM. The EDEN Mother-Child Cohort. https://eden.vjf.inserm.fr/

15 Tzoumaka-Bakoula C. The Greek national perinatal survey: I: design, methodology, case ascertainment. Paediatr Perinat Epidemiol 1987;1:43-55.

16 Palili A, Kolaitis G, Vassi I, et al. Inattention, hyperactivity, impulsivityepidemiology and correlations: a nationwide greek study from birth to 18 years. J Child Neurol 2011:26:199-204.

17 Porta D, Forastiere F, Di Lallo D, et al. Enrolment and follow-up of a birth cohort in Rome. Epidemiol Prev 2007:31:303-8.

18 Porta D, Fantini MP, on behalf of the GASPII and CO.N.ER Study Groups. Prospective cohort studies of newborns in Italy to evaluate the role of environmental and genetic characteristics on common childhood disorders. Ital J Pediatr 2006;32:350-7.

19 van Eijsden M, Vrijkotte TG, Gemke RJ, et al. Cohort profile: the Amsterdam Born Children and their Development (ABCD) study. Int J Epidemiol 2011;40:1176-86.

20 Eggesbo M, Stigum H, Longnecker MP, et al. Levels of hexachlorobenzene (HCB) in breast milk in relation to birth weight in a Norwegian cohort. Environ Res 2009;109:559-66.

21 Pinto E, Barros $\mathrm{H}$, dos Santos Silva I. Dietary intake and nutritional adequacy prior to conception and during pregnancy: a follow-up study in the north of Portugal. Public Health Nutr 2009:12:922-31.

22 Guxens M, Ballester F, Espada M, et al. Cohort Profile: the INMA-INfancia y Medio Ambiente-(Environment and Childhood) Project. Int J Epidemiol 2012:41:930-40.

23 Huus K, Ludvigsson JF, Enskar K, et al. Exclusive breastfeeding of Swedish children and its possible influence on the development of obesity: a prospective cohort study. BMC Pediatr 2008;8:42.
24 Plewis I, Calderwood L, Hawkes D, et al. The Millennium Cohort Study: Technical Report on Sampling. 4th edn. London: Bedford Group for Lifecourse and Statistical Studies, Institute of Education, University of London, 2007.

25 The University of Illinois at Chicago. Ukraine: Family and Children of Ukraine (FCOU). http://uic.edu/sph/glakes/research/ukraine_children.html (accessed 27 Jan 2015).

26 Organisation for Economic Co-operation and Development. Classifying Educational Programmes: Manual for ISCED-97 Implementation in OECD Countries. 1999.

27 Alexander GR, Himes JH, Kaufman RB, et al. A United States national reference for fetal growth. Obstet Gynecol 1996;87:163-8.

28 Committee on Understanding Premature Birth and Assuring Healthy Outcomes, IOM. Preterm Birth: Causes, Consequences, and Prevention. Washington, DC: Institute of Medicine (IOM), 2007.

29 World Health Organisation Regional Office for Europe. European health for all database. http://www.euro.who.int/en/data-and-evidence/databases/ european-health-for-all-database-hfa-db (accessed 27 Jan 2015).

30 Mackenbach JP, Kunst AE. Measuring the magnitude of socio-economic inequalities in health: an overview of available measures illustrated with two examples from Europe. Soc Sci Med 1997;44:757-71.

31 Kunst AE, Bos V, Mackenbach JP, EU Working Group on Socio-economic Inequalities in Health. Monitoring socio-economic inequalities in health in the European Union: guidelines and illustrations. The Netherlands: Department of Public Health, Erasmus University Rotterdam, 2001.

32 Harper S, Lynch J. Methods for measuring cancer disparities: using data relevant to healthy people 2010 cancer-related objectives. Bethesda, MD: National Cancer Institute, 2005.

33 Bross IDJ. How to use ridit analysis. Biometrics 1958;14:18-38.

34 Harris R, Bradburn M, Deeks J, et al. Metan: fixed- and random-effects metaanalysis. Stata J 2008:8:3-28.

35 Kunst AE, Mackenbach JP. The size of mortality differences associated with educational level in nine industrialized countries. Am J Public Health 1994;84 932-7.

36 Rosengren A, Subramanian SV, Islam S, et al. Education and risk for acute myocardial infarction in 52 high, middle and low-income countries: INTERHEART case-control study. Heart 2009;95:2014-22.

37 Ross CE, Wu CL. Education, age, and the cumulative advantage in health. J Health Soc Behav 1996;37:104-20.

38 Graham H. Introduction: The Challenge of Health Inequalities. In: Graham H, ed. Understanding Health Inequalities. 2nd edn. Berkshire: Open University Press, 2009:1-21.

39 Hemming K, Hutton JL, Glinianaia SV, et al. Differences between European birthweight standards: impact on classification of 'small for gestational age'. Dev Med Child Neurol 2006:48:906-12.

40 Lack N, Zeitlin J, Krebs L, et al. Methodological difficulties in the comparison of indicators of perinatal health across Europe. Eur J Obstet Gynecol Reprod Biol 2003;111(Suppl 1):S33-44 